Research Article

\title{
Energy Levels in Nanowires and Nanorods with a Finite Potential Well
}

\author{
G. Gulyamov $\mathbb{D}^{1},{ }^{1}$ A. G. Gulyamov $\mathbb{D}^{2},{ }^{2}$ A. B. Davlatov $\mathbb{D}^{2},{ }^{2}$ and Kh. N. Juraev $\mathbb{D}^{2}$ \\ ${ }^{1}$ Namangan Engineering Construction Institute, 12 Islam Karimov Street, Namangan 160103, Uzbekistan \\ ${ }^{2}$ Physical-Technical Institute, Uzbek Academy of Sciences, Chingiz Aytmatov Street, 2 “B”, Tashkent 100084, Uzbekistan \\ Correspondence should be addressed to Kh. N. Juraev; khimmatali@gmail.com
}

Received 19 July 2020; Revised 15 October 2020; Accepted 19 October 2020; Published 7 November 2020

Academic Editor: Kamakhya Prasad Ghatak

Copyright (c) 2020 G. Gulyamov et al. This is an open access article distributed under the Creative Commons Attribution License, which permits unrestricted use, distribution, and reproduction in any medium, provided the original work is properly cited.

The energy of electrons and holes in cylindrical quantum wires with a finite potential well was calculated by two methods. An analytical expression is approximately determined that allows one to calculate the energy of electrons and holes at the first discrete level in a cylindrical quantum wire. The electron energy was calculated by two methods for cylindrical layers of different radius. In the calculations, the nonparabolicity of the electron energy spectrum is taken into account. The dependence of the effective masses of electrons and holes on the radius of a quantum wires is determined. An analysis is made of the dependence of the energy of electrons and holes on the internal and external radii, and it is determined that the energy of electrons and holes in cylindrical layers with a constant thickness weakly depends on the internal radius. The results were obtained for the InP/ InAs heterostructures.

\section{Introduction}

In recent years, a lot of work has been done on calculating the energy of electrons and holes in quantum wells based on InP/InAs/InP heterostructures, due to the fact that today, for the creation of new-generation devices, it largely depends on semiconductor nanostructures.

In $[1,2]$, various technologies for growing quantum wires were presented, and nanowires of various sizes were obtained. In [3], core-shell nanowires were experimentally obtained, and it was shown that the cross-sectional area is in the shape of a hexagon. Optical properties of InAs/InP-based quantum wires were studied by photoluminesce spectroscopy [4]. The optical properties of core-multishell nanowires based on InP/InAs/InP were experimentally studied in [5-8]. The relaxation time of electron spin in semiconductor quantum wires has been experimentally investigated.

In type III-V semiconductors and in heterostructures based on them, electron dispersion is strongly nonparabolic; the Kane model was used to study the spectrum of charge carriers of these materials [9]. In [10], the energy spectrum of an electron with a nonparabolic dispersion law in quantum wires with a rectangular cross-sectional shape was theoretically investigated. The authors of $[11,12]$ also theoretically studied the energy spectrum of an electron with a nonparabolic dispersion law in quantum wires, but with a hexagonal and triangular cross-sectional shape. The influence of electronic, polaron, and coulomb interactions on energy states in quantum nanowires was studied [13-16]. The I-V characteristic of nanowires was considered taking into account the tunneling of electrons in quantum states $[17,18]$. The effect of temperature on the energy levels $[19,20]$ and optical absorption [21] of quantum dots are studied. The energy levels of InAs/InP quantum dots have been studied [22]. In [23-28], to determine the energy spectrum and wave function of an electron in quantum wires with a rectangular cross-sectional shape, solutions of the Schrödinger equation were obtained using various mathematical methods. The effect of an electric field on the energy spectrum of a rectangular quantum wire is investigated [29].

The authors of [30-33] obtained analytical solutions of the Schrödinger equation for cylindrical quantum wires with a finite potential and a parabolic dispersion law. The solution of the Schrödinger equation is obtained by the finite 
difference method (shooting method) for rectangular [34] and cylindrical $[35,36]$ quantum wires.

In this paper, two methods are used to calculate the energy of electrons and holes for a cylindrical quantum wire and a quantum nanorod with a finite potential well. In this case, the nonparabolicity of the dispersion of electrons and holes is taken into account. The relationship between the effective mass of charge carriers and the radius of a quantum wire is determined.

\section{An Analytical Method for Calculating the Electron Energy in a Cylindrical Quantum Wire with a Finite Height of the Potential Well}

Figure 1 shows the geometric and potential diagram of a cylindrical quantum wire with a finite potential well. The potential energy of an electron of a cylindrical quantum wire with a finite depth has the form:

$$
U(r)=U(\rho)= \begin{cases}0, & 0 \leq \rho \leq R, \\ W, & \rho>R .\end{cases}
$$

The Schrödinger equation in a cylindrical coordinate system is as follows:

$$
\begin{aligned}
& -\frac{\hbar^{2}}{2}\left(\frac{1}{\rho} \frac{\partial}{\partial \rho} \frac{\rho}{m(\rho)} \frac{\partial}{\partial \rho}+\frac{1}{\rho^{2}} \frac{\partial^{2}}{\partial \phi^{2}}+\frac{\partial^{2}}{\partial z^{2}}\right) f(r) \\
& +U(r) f(r)=\operatorname{Ef}(r) .
\end{aligned}
$$

We seek a solution to this equation in the following form:

$$
f(r)=e^{i k_{z} z} e^{i l \phi} \psi(\rho),
$$

Here, the parameters $k_{x}$ and $l$ are independent of coordinates. Therefore, equation (2) will be solved for the radial wave function. Thus, equation (2) in region $0 \leq \rho \leq R$ for the radial wave function $\psi(\rho)$ takes the following form:

$$
\frac{\partial^{2} \psi(\rho)}{\partial \rho^{2}}+\frac{1}{\rho} \frac{\partial \psi(\rho)}{\partial \rho}+\left(k_{A}^{2}-\left(\frac{l^{2}}{\rho^{2}}\right)\right) \psi(\rho)=0, \quad 0 \leq \rho \leq R,
$$

where

$$
\begin{aligned}
\xi & =k_{A} \rho, \\
k_{A} & =\sqrt{\frac{2 m_{A}}{\hbar^{2}} E-k_{z}^{2} .}
\end{aligned}
$$

In this case, equation (4) takes the following form:

$$
\xi^{2} \frac{\partial^{2} \psi(\rho)}{\partial \xi^{2}}+\xi \frac{\partial \psi(\rho)}{\partial \xi}+\left(\xi^{2}-l^{2}\right) \psi(\rho)=0
$$

The general solution to this equation is in the form of a linear combination of the Bessel function $J_{l}(\xi)$ and the Neumann function $N_{l}(\xi)$ of the $l$-th order [37]:

$$
\psi_{1}(\rho)=A_{1} J_{l}\left(k_{A} \rho\right)+B_{1} N_{l}\left(k_{A} \rho\right), \quad 0 \leq \rho \leq R .
$$
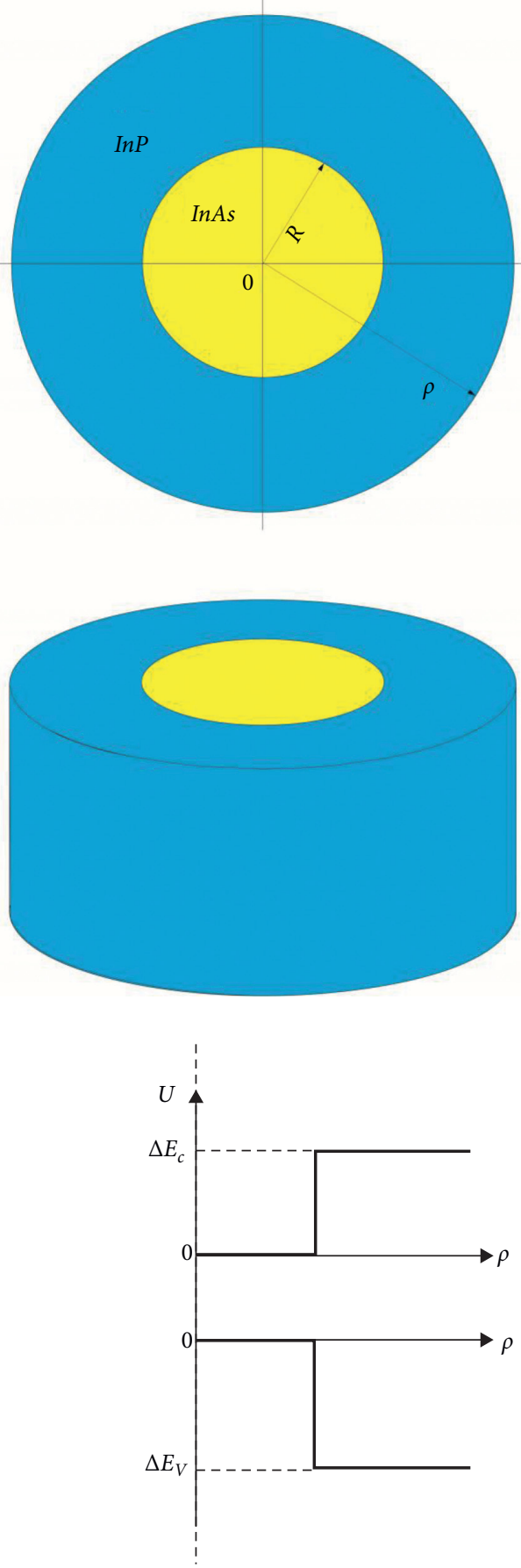

Figure 1: Geometric and potential diagram of a cylindrical quantum wire with a finite potential well.

Equation (2) for the radial wave function $\psi(\rho)$ in region $\rho>R$ takes the following form:

$$
\zeta^{2} \frac{\partial^{2} \psi}{\partial \zeta^{2}}+\zeta \frac{\partial \psi}{\partial \zeta}+\left(-\zeta^{2}-M^{2}\right) \psi=0, \quad \rho>R
$$

where 


$$
\begin{aligned}
\zeta & =\gamma_{B} \rho, \\
\gamma_{B} & =\sqrt{\frac{2 m_{B}}{\hbar^{2}}(W-E)+k_{z}^{2}} .
\end{aligned}
$$

The solution of equation (8) gives a linear combination of the imaginary argument $I_{l}(\zeta)$ of the Bessel function and the Macdonald function $K_{l}(\zeta)$ of the $l$-th order [37]:

$$
\psi_{2}(\rho)=A_{2} K_{l}\left(\gamma_{B} \rho\right)+B_{2} I_{l}\left(\gamma_{B} \rho\right), \quad \rho>R .
$$

Therefore, $\psi(\rho)$ for a radial wave function is appropriate for the following:

$$
\psi(\rho)= \begin{cases}A_{1} J_{l}\left(k_{A} \rho\right)+B_{1} N_{l}\left(k_{A} \rho\right), & 0 \leq \rho \leq R, \\ A_{2} K_{l}\left(\gamma_{B} \rho\right)+B_{2} I_{l}\left(\gamma_{B} \rho\right), & \rho>R .\end{cases}
$$

If we take into account that the wave function inside the cylinder is finite and equal to zero at an infinite distance from the center of the cylinder, expression (11) takes the following form:

$$
\psi(\rho)= \begin{cases}A_{1} J_{l}\left(k_{A} \rho\right), & 0 \leq \rho \leq R \\ A_{2} K_{l}\left(\gamma_{B} \rho\right), & \rho>R\end{cases}
$$

Here, $A_{1}$ and $A_{2}$ are constant values. We select relation $A_{1} / A_{2}$ in such a way that the following boundary conditions are satisfied:

$$
\begin{gathered}
\left.\psi_{1}(\rho)\right|_{\rho=R}=\left.\psi_{2}(\rho)\right|_{\rho=R}, \\
\left.\frac{1}{m_{A}} \frac{d \psi_{1}(\rho)}{d \rho}\right|_{\rho=R}=\left.\frac{1}{m_{B}}\left(\frac{d \psi_{2}(\rho)}{d \rho}\right)\right|_{\rho=R} .
\end{gathered}
$$

From (12) and (13), we obtain the following transcendental equation:

$$
\frac{J_{l}^{\prime}\left(k_{A} R\right) K_{l}\left(\gamma_{B} R\right)}{J_{l}\left(k_{A} R\right) K_{l}^{\prime}\left(\gamma_{B} R\right)}=\frac{m_{A}}{m_{B}} .
$$

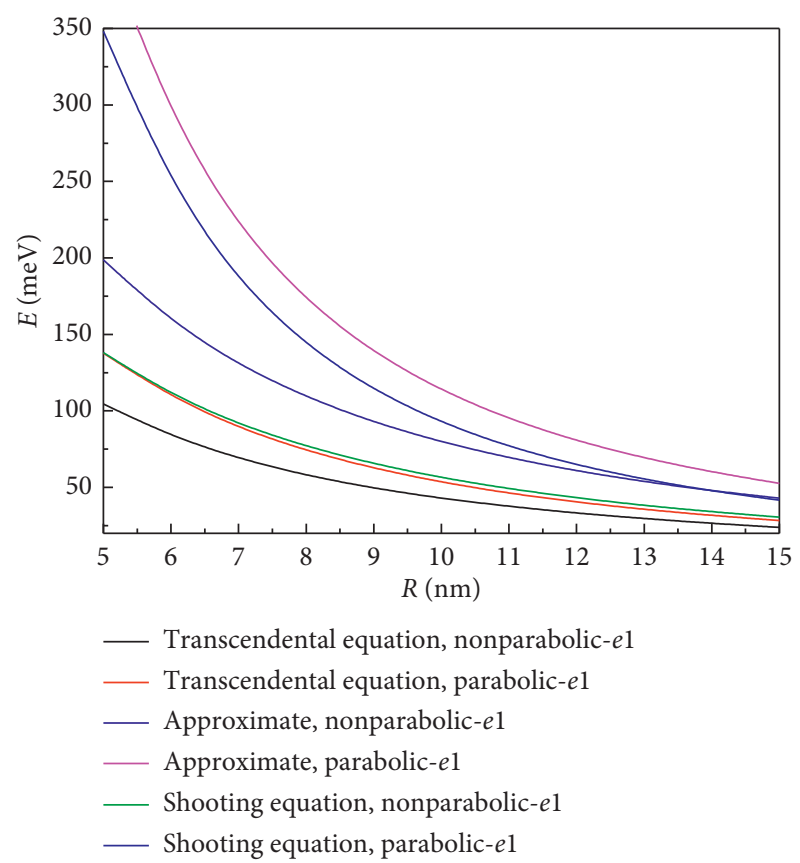

Figure 2: The dependence of energy on the radius of a cylindrical nanowire for electrons and holes with the parabolic and nonparabolic dispersion law.

Solving the transcendental equation (14), we can determine the electron energy in a cylindrical quantum wire.

When the argument is too small under condition $l=0$, expanding the imaginary argument of the Bessel function $I_{l}(\zeta)$ and MacDonald $K_{l}(\zeta)$ in a row, we get the first terms and an analytical formula for calculating the energy:

$$
E_{0,1}=\frac{4 \hbar^{2}}{m_{A} R^{2}} \frac{\sqrt{\left(2 m_{B} W / \hbar^{2}\right)} R K_{0}\left(\sqrt{\left(2 m_{B} W / \hbar^{2}\right)} R\right)+2 K_{1}\left(\sqrt{\left(2 m_{B} W / \hbar^{2}\right)} R\right)}{\sqrt{\left(2 m_{B} W / \hbar^{2}\right)} R K_{0}\left(\sqrt{\left(2 m_{B} W / \hbar^{2}\right)} R\right)+4 K_{1}\left(\sqrt{\left(2 m_{B} W / \hbar^{2}\right)} R\right)} .
$$

If we take into account (5) and (9), expression (15) gives very close results to the exact results of equation (14) when calculating the energy of electrons and holes in cylindrical nanowires with larger radii (Figures 2-4).

\section{The Analytical Method for Calculating the Electron Energy in a Cylindrical Quantum Nanorod with a Finite Height of the Potential Well}

Figure 5 shows the geometric and potential diagram of a cylindrical quantum nanorod with a finite potential well. Let the potential energy of an electron in a cylindrical quantum nanorod with a finite depth be given by the following expression:

$$
U(r)=U(\rho)= \begin{cases}W, & 0<\rho<R_{1} \\ 0, & R_{1} \leq \rho \leq R_{2} \\ W, & R_{2}<\rho\end{cases}
$$

The Schrödinger equation in a cylindrical coordinate system in this case is as follows: 


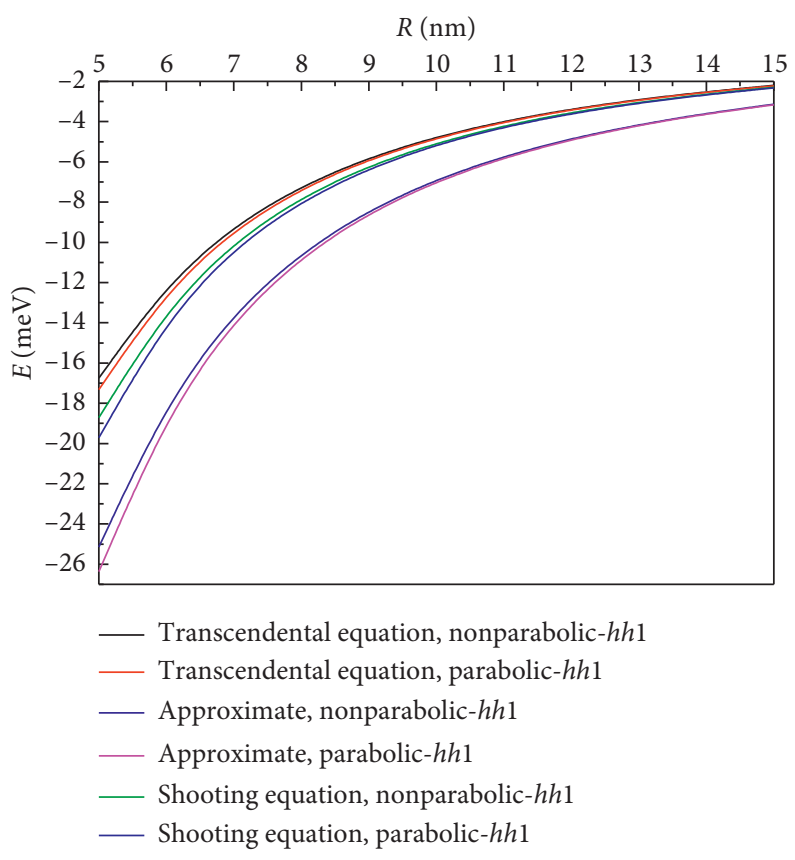

FIgURe 3: The dependence of the energy of a heavy hole on the radius of a cylindrical quantum wire for the parabolic and nonparabolic dispersion law.

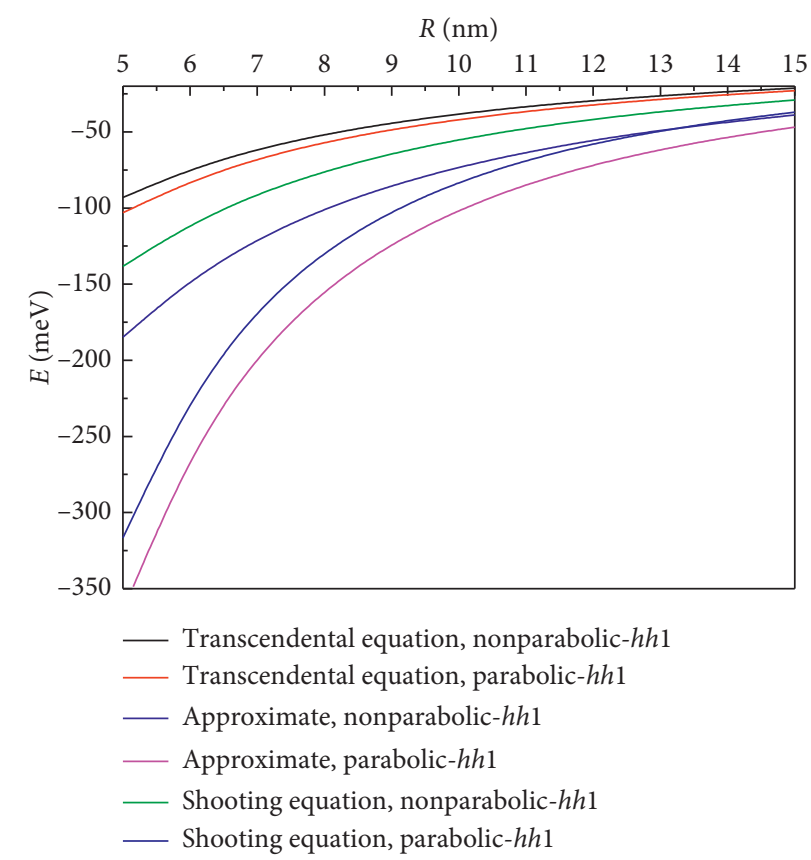

FIGURE 4: The dependence of the energy of a light hole on the radius of a cylindrical quantum wire for the parabolic and nonparabolic dispersion law. 
$-\frac{\hbar^{2}}{2}\left(\frac{1}{\rho} \frac{\partial}{\partial \rho} \frac{\rho}{m(\rho)} \frac{\partial}{\partial \rho}+\frac{1}{\rho^{2}} \frac{\partial^{2}}{\partial \phi^{2}}+\frac{\partial^{2}}{\partial z^{2}}\right) f(r)+U(r) f(r)=\operatorname{Ef}(r)$

The solution to the radial part of the equation will be

$$
\psi(\rho)= \begin{cases}A_{1} K_{l}\left(\gamma_{B} \rho\right)+B_{1} I_{l}\left(\gamma_{B} \rho\right), & 0<\rho<R_{1}, \\ A_{2} J_{l}\left(k_{A} \rho\right)+B_{2} N_{l}\left(k_{A} \rho\right), & R_{1} \leq \rho \leq R_{2}, \\ A_{3} K_{l}\left(\gamma_{B} \rho\right)+B_{3} I_{l}\left(\gamma_{B} \rho\right), & R_{2}<\rho .\end{cases}
$$

If we take into account that the wave function inside a cylindrical quantum nanorod is finite and equal to zero at an infinite distance from the center of the quantum nanorod, then expression (18) takes the following form:

$$
\psi(\rho)= \begin{cases}B_{1} I_{l}\left(\gamma_{B} \rho\right), & 0<\rho<R_{1}, \\ A_{2} J_{l}\left(k_{A} \rho\right)+B_{2} N_{l}\left(k_{A} \rho\right), & R_{1} \leq \rho \leq R_{2}, \\ A_{3} K_{l}\left(\gamma_{B} \rho\right), & R_{2}<\rho .\end{cases}
$$

For the continuity of the wave function, the following conditions must be met:

$$
\begin{gathered}
\psi_{1}(\rho)_{\mid \rho=R_{1}}=\left.\psi_{2}(\rho)\right|_{\rho=R_{1}}, \\
\left.\frac{1}{m_{B}} \frac{d \psi_{1}(\rho)}{d \rho}\right|_{\rho=R_{1}}=\left.\frac{1}{m_{A}} \frac{d \psi_{2}(\rho)}{d \rho}\right|_{\rho=R_{1}}, \\
\left.\psi_{2}(\rho)\right|_{\rho=R_{2}}=\left.\psi_{3}(\rho)\right|_{\rho=R_{2}}, \\
\left.\frac{1}{m_{A}} \frac{d \psi_{2}(\rho)}{d \rho}\right|_{\rho=R_{2}}=\left.\frac{1}{m_{B}} \frac{d \psi_{3}(\rho)}{d \rho}\right|_{\rho=R_{2}} .
\end{gathered}
$$

Applying boundary conditions (20) to expression (19), we obtain the following system of equations:

$$
\left\{\begin{array}{l}
B_{1} I_{l}\left(\gamma_{B} R_{1}\right)=A_{2} J_{l}\left(k_{A} R_{1}\right)+B_{2} N_{l}\left(k_{A} R_{1}\right), \\
\frac{1}{m_{B}} B_{1} I_{l}^{\prime}\left(\gamma_{B} R_{1}\right)=\frac{1}{m_{A}}\left(A_{2} J_{l}^{\prime}\left(k_{A} R_{1}\right)+B_{2} N_{l}^{\prime}\left(k_{A} R_{1}\right)\right), \\
A_{2} J_{l}\left(k_{A} R_{2}\right)+B_{2} N_{l}\left(k_{A} R_{2}\right)=A_{3} K_{l}\left(\gamma_{B} R_{2}\right), \\
\frac{1}{m_{A}}\left(A_{2} J_{l}^{\prime}\left(k_{A} R_{2}\right)+B_{2} N_{l}^{\prime}\left(k_{A} R_{2}\right)\right)=\frac{1}{m_{B}} A_{3} K_{l}^{\prime}\left(\gamma_{B} R_{2}\right) .
\end{array}\right.
$$

Solving the system of equation (21), we obtain the following transcendental equation:
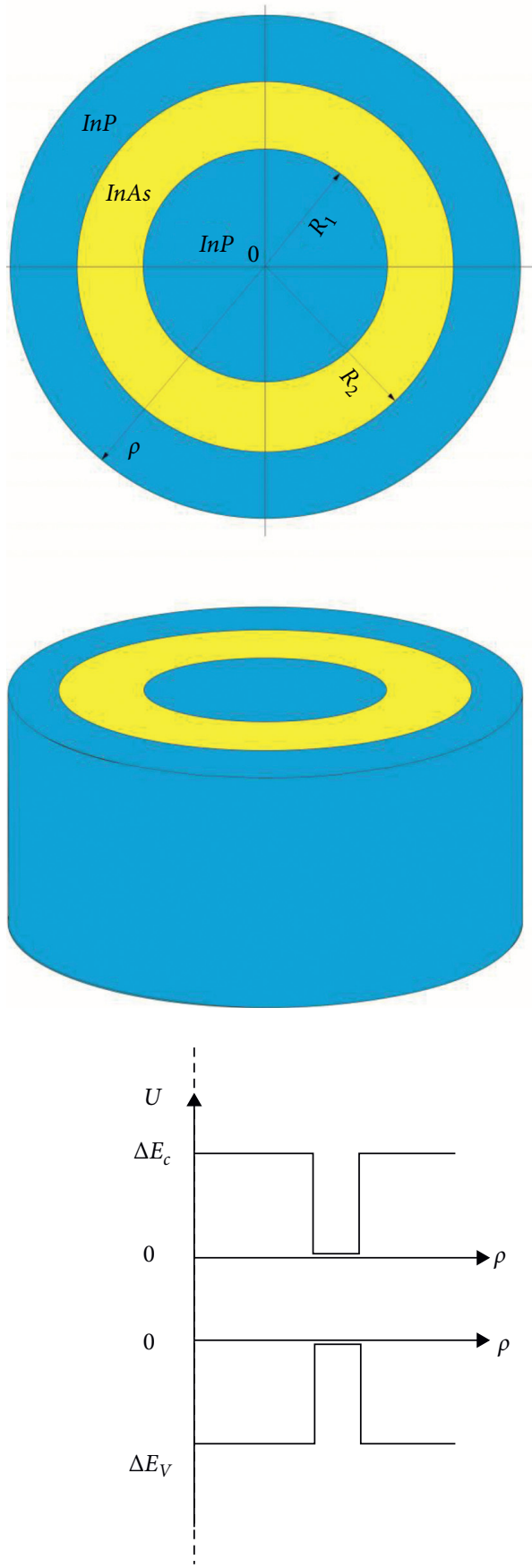

FIGURE 5: The geometric and potential scheme of a cylindrical quantum nanorod with a finite potential well.

$$
\frac{\left(m_{A} / m_{B}\right) J_{l}\left(k_{A} R_{1}\right) I_{l}^{\prime}\left(\gamma_{B} R_{1}\right)-J_{l}^{\prime}\left(k_{A} R_{1}\right) I_{l}\left(\gamma_{B} R_{1}\right)}{\left(m_{A} / m_{B}\right) J_{l}\left(k_{A} R_{2}\right) K_{l}^{\prime}\left(\gamma_{B} R_{2}\right)-J_{l}^{\prime}\left(k_{A} R_{2}\right) K_{l}\left(\gamma_{B} R_{2}\right)}=\frac{N_{l}^{\prime}\left(k_{A} R_{1}\right) I_{l}\left(\gamma_{B} R_{1}\right)-\left(m_{A} / m_{B}\right) N_{l}\left(k_{A} R_{1}\right) I_{l}^{\prime}\left(\gamma_{B} R_{1}\right)}{N_{l}^{\prime}\left(k_{A} R_{2}\right) K_{l}\left(\gamma_{B} R_{2}\right)-\left(m_{A} / m_{B}\right) N_{l}\left(k_{A} R_{2}\right) K_{l}^{\prime}\left(\gamma_{B} R_{2}\right)} .
$$




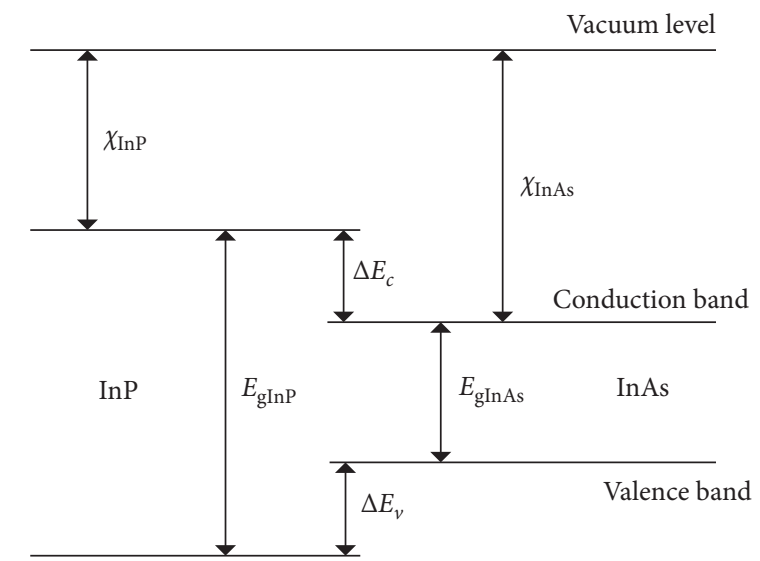

Figure 6: The band diagram of the InP/InAs heterostructure.

Transcendental equation (22) allows us to calculate the energy of electrons and holes in cylindrical nanolayers with a finite potential well.

\section{The Shooting Method for Calculating the Electron Energy in a Cylindrical Quantum Wire and in a Quantum Nanorod with a Finite Height of a Potential Well}

Given the effective mass, the Schrödinger equation will be as follows:

$$
-\frac{\hbar^{2}}{2 \rho} \frac{\partial}{\partial \rho} \frac{\rho}{m(\rho)} \frac{\partial}{\partial \rho} \psi(\rho)+V(\rho) \psi(\rho)=E \psi(\rho) .
$$

Equation (23) is used for the parabolic dispersion law, and it is solved by the finite difference method-the
Shooting method [38]. We will try to solve equation (23) by the same method for the nonparabolic dispersion law. In this case, we represent the wave function and the derivative of the effective mass in the following form:

$$
\begin{gathered}
\frac{d \psi}{d \rho} \approx \frac{\psi(\rho+\delta \rho)-\psi(\rho-\delta \rho)}{2 \delta \rho}, \\
\frac{d^{2} \psi}{d \rho^{2}} \approx \frac{\psi(\rho+\delta \rho)-2 \psi(\rho)+\psi(\rho-\delta \rho)}{(\delta \rho)^{2}}, \\
\frac{d m}{d \rho} \approx \frac{m(\rho+\delta \rho)-m(\rho-\delta \rho)}{2 \delta \rho} .
\end{gathered}
$$

Using (23)-(26), we obtain the following:

$$
\begin{aligned}
& \rho m(\rho) \frac{\psi(\rho+\delta \rho)-2 \psi(\rho)+\psi(\rho-\delta \rho)}{(\delta \rho)^{2}}-\frac{\psi(\rho+\delta \rho)-\psi(\rho-\delta \rho)}{2 \delta \rho}\left(\rho\left(\frac{m(\rho+\delta \rho)-m(\rho-\delta \rho)}{2 \delta \rho}\right)-m(\rho)\right), \\
& =\frac{2 \rho(m(\rho))^{2}}{\hbar^{2}}[V(\rho)-E] \psi(\rho) .
\end{aligned}
$$

From here, we get

$\psi(\rho+\delta \rho)=\frac{8\left\{\left((\delta \rho m(\rho))^{2} / \hbar^{2}\right)[V(\rho)-E]+m(\rho)\right\}}{\{2 m(\rho)(2+(\delta \rho / \rho))-m(\rho+\delta \rho)+m(\rho-\delta \rho)\}} \psi(\rho)-\frac{\{2 m(\rho)(2-(\delta \rho / \rho))+m(\rho+\delta \rho)-m(\rho-\delta \rho)\}}{\{2 m(\rho)(2+(\delta \rho / \rho))-m(\rho+\delta \rho)+m(\rho-\delta \rho)\}} \psi(\rho-\delta \rho)$

If the values of $\psi(\rho-\delta \rho)$ and $\psi(\rho)$ are known for wave functions, then using (28), it is possible to determine the value of $\psi(\rho+\delta \rho)$ for an arbitrary energy. In order to calculate the wave function and energy of electrons and holes, it is necessary to take into account the following three boundary conditions:

$$
\begin{gathered}
\psi(\infty) \longrightarrow 0, \\
\psi(0)=1, \\
\psi(\delta \rho)=1 .
\end{gathered}
$$


TABLE 1: Material parameters of InAs and InP.

\begin{tabular}{|c|c|c|}
\hline Parameter & InAs & InP \\
\hline \multirow{5}{*}{$E_{g}(e V)$} & $0.35^{[39]}$ & $1.35^{[39,44,45]}$ \\
\hline & $0.36^{[44,46]}$ & $1.424^{[47]}$ \\
\hline & $0.417^{[47,48]}$ & $1.423^{[48]}$ \\
\hline & $0.42^{[48]}$ & $1.42^{[48,49]}$ \\
\hline & $0.418^{[49]}$ & \\
\hline \multirow{3}{*}{$m_{e} / m_{0}$} & $0.022^{[39]}$ & $0.077^{[39,47,50]}$ \\
\hline & $0.023^{[44,47,48,50]}$ & $0.08^{[44]}$ \\
\hline & $0.024^{[48]}$ & $0.079^{[48,49]}$ \\
\hline \multirow{4}{*}{$m_{h h} / m_{0}$} & $0.41^{[39,48]}$ & $0.6^{[39]}$ \\
\hline & $0.6^{[44]}$ & $0.85^{[44]}$ \\
\hline & $0.34^{[50]}$ & $0.65^{[48]}$ \\
\hline & & $0.472^{[50]}$ \\
\hline \multirow{3}{*}{$m_{l h} / m_{0}$} & $0.026^{[39]}$ & $0.12^{[39,48]}$ \\
\hline & $0.027^{[44,50]}$ & $0.098^{[44]}$ \\
\hline & $0.025^{[48]}$ & $0.096^{[50]}$ \\
\hline \multirow{2}{*}{$\chi(e V)$} & $4.92^{[39]}$ & $4.38^{[39]}$ \\
\hline & $4.9^{[45]}$ & $4.4^{[45]}$ \\
\hline \multirow{2}{*}{$2 m_{0} P^{2} / \hbar^{2}(e V)$} & $22.2^{[39]}$ & $20.4^{[39]}$ \\
\hline & $21.5^{[47,48]}$ & $20.7^{[47,48]}$ \\
\hline \multirow{3}{*}{$\begin{array}{l}2 m_{n} P^{2} / \hbar^{2}(e V) \\
\Delta_{0}(e V)\end{array}$} & $0.5^{[48]}$ & $1.46^{[48]}$ \\
\hline & $0.38^{[39,47,49]}$ & $0.11^{[39,49]}$ \\
\hline & $0.39^{[48]}$ & $0.108^{[47,48]}$ \\
\hline
\end{tabular}

TABLE 2: Material parameters of InAs and InP.

\begin{tabular}{lccccccccccc}
\hline Material parameters & $\begin{array}{l}E_{g} \\
(\mathrm{eV})\end{array}$ & $\frac{m_{e}}{m_{0}}$ & $\frac{m_{h h}}{m_{0}}$ & $\frac{m_{l h}}{m_{0}}$ & $\begin{array}{c}\chi \\
(\mathrm{eV})\end{array}$ & $\begin{array}{c}\alpha_{e} \\
(1 / \mathrm{eV})\end{array}$ & $\begin{array}{c}\alpha_{h h} \\
(1 / \mathrm{eV})\end{array}$ & $\begin{array}{c}\alpha_{l h} \\
(1 / \mathrm{eV})\end{array}$ & $\begin{array}{c}\Delta_{0} \\
(\mathrm{eV})\end{array}$ & $\begin{array}{c}\Delta E_{C} \\
(\mathrm{eV})\end{array}$ & $\begin{array}{l}\Delta E_{V} \\
(\mathrm{eV})\end{array}$ \\
\hline InAs & 0.36 & 0.023 & 0.41 & 0.026 & 4.91 & 2.65 & 0.97 & 2.63 & 0.38 & 0.52 & 0.47 \\
InP & 1.35 & 0.077 & 0.65 & 0.096 & 4.39 & 0.63 & 0.09 & 0.6 & 0.11 & \\
\hline
\end{tabular}

$E_{\mathrm{g}}$ is the band gap, $m_{\mathrm{e}} / m_{0}$ is the effective mass of the electron in the conduction band, $m_{\mathrm{lh}} / m_{0}$ and $m_{\mathrm{hh}} / \mathrm{m}_{0}$ are the effective masses of light and heavy holes in the valence band, $\chi$ is the electron affinity, $\alpha_{\mathrm{i}}$ is the nonparabolicity coefficient, and $\Delta_{0}$ is spin-orbit interaction.

\section{Analysis of the Results}

It is known that the lattice constant for $\mathrm{InP}$ is $0.5869 \mathrm{~nm}$, and this value is close to the lattice constant for InAs $0.6058 \mathrm{~nm}$ [39]. This allows you to get the perfect heterostructures using these materials. Figure 6 shows the band diagram of the InP/ InAs based heterostructures. To take into account the nonparabolicity of the zone, we use the Kane model [9]. Various approximations for the effective mass are given in $[10,40-43]$; we will use the following expression for the effective mass:

$$
\begin{aligned}
m_{\mathrm{InPi}}(E) & =m_{0 / \mathrm{InPi}}\left(1+\alpha_{\mathrm{InPi}}(E-V(\rho))\right), \\
\alpha_{\mathrm{InPi}} & =\frac{1}{E_{\mathrm{gInP}}}\left(1-\frac{m_{i}}{m_{0}}\right)^{2} \\
\alpha_{\mathrm{In} A s i} & =\frac{1}{E_{\mathrm{gInAs}}}\left(1-\frac{m_{i}}{m_{0}}\right)^{2}
\end{aligned}
$$

where $i=e, h h, l h$.

Table 1 shows the necessary parameters for InAs and InP obtained by various authors. For our calculations, we will choose the parameters shown in Table 2.

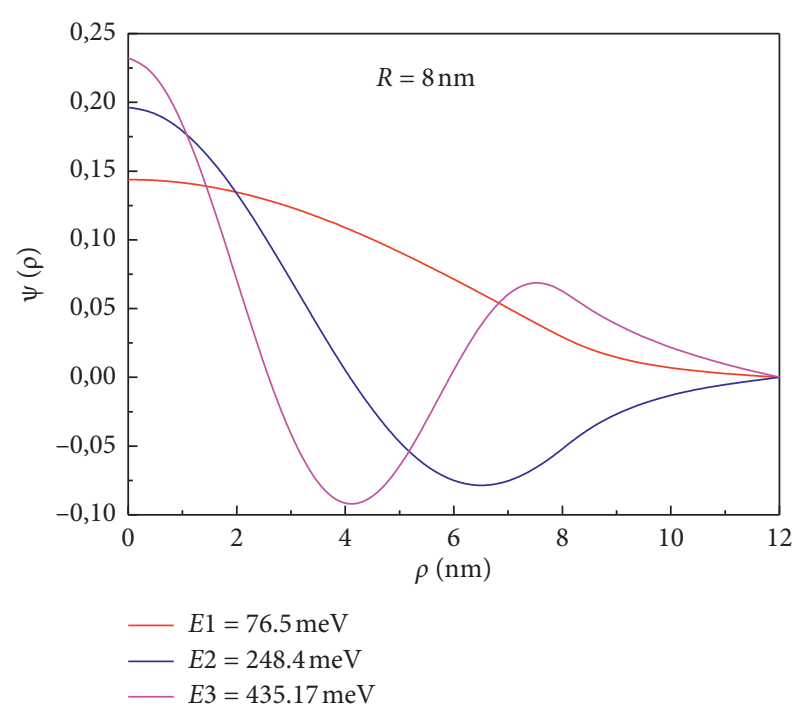

FIgURE 7: The dependence of the radial wave function of an electron on the radius of a cylindrical quantum wire with a finite potential. 


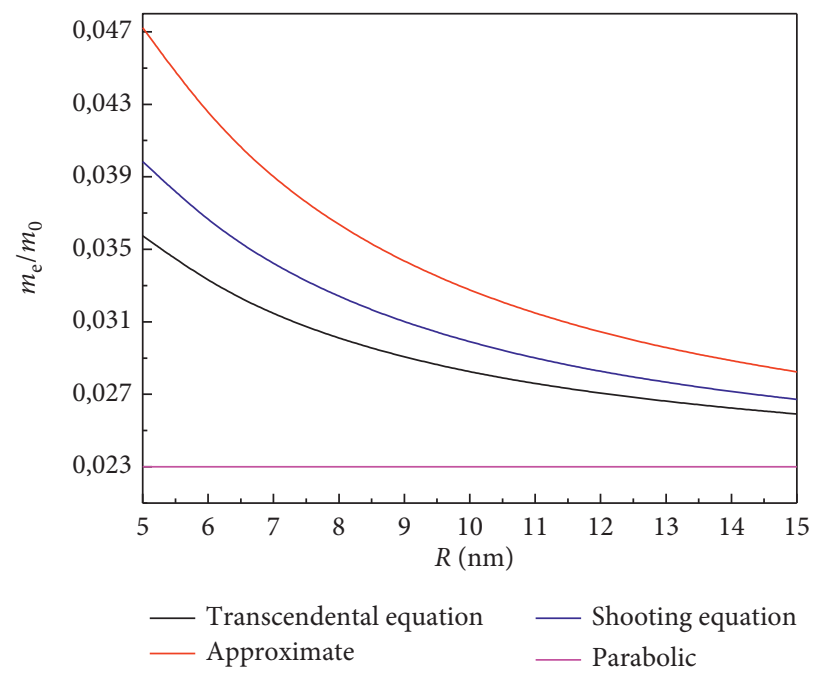

FIGURE 8: The dependence of the effective electron mass on the radius of a cylindrical quantum wire with a finite potential.

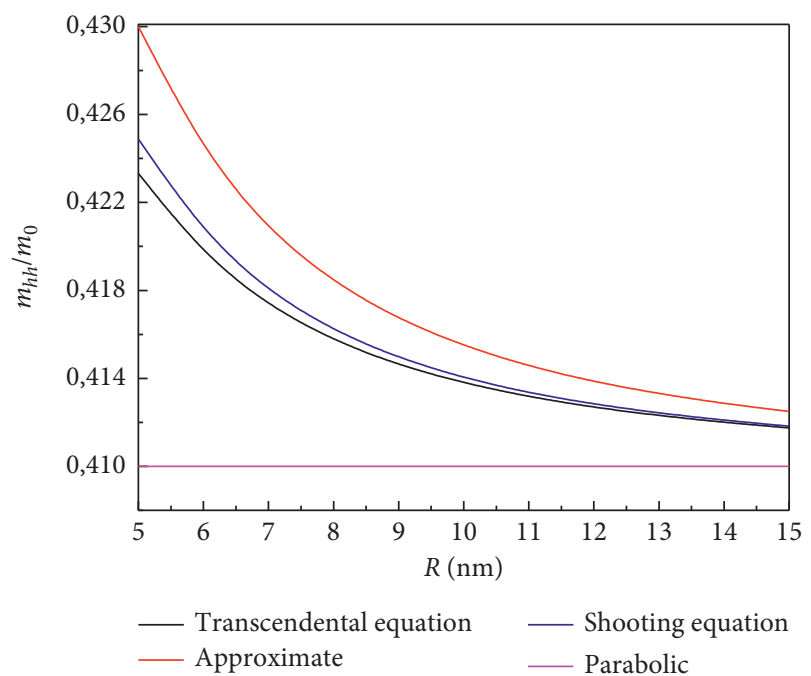

FIGURE 9: The dependence of the effective mass of a heavy hole on the radius of a cylindrical quantum wire with a finite potential.

A numerical solution of transcendental equation (14) determines the wave functions and the energy of electrons and holes. By an approximate solution of this equation, equation (15) is obtained. For large radii, the solution of equation (15) gives results that are close to the exact results of equation (14). The energy and wave function of the particles are calculated using expression (28), which is obtained by the finite difference method. The calculations were performed taking into account the nonparabolicity of the zones using expression (30). The dependence of the energy on the radius of a cylindrical nanowire for electrons and holes is shown taking into account the parabolic and nonparabolic zones (Figures 2-4). Figure 7 shows the radial wave function of the electron. Usually, the electron wave function as $\rho \longrightarrow \infty$ is equal to zero. If at the size of the potential well $R$, we choose that the wave function at a

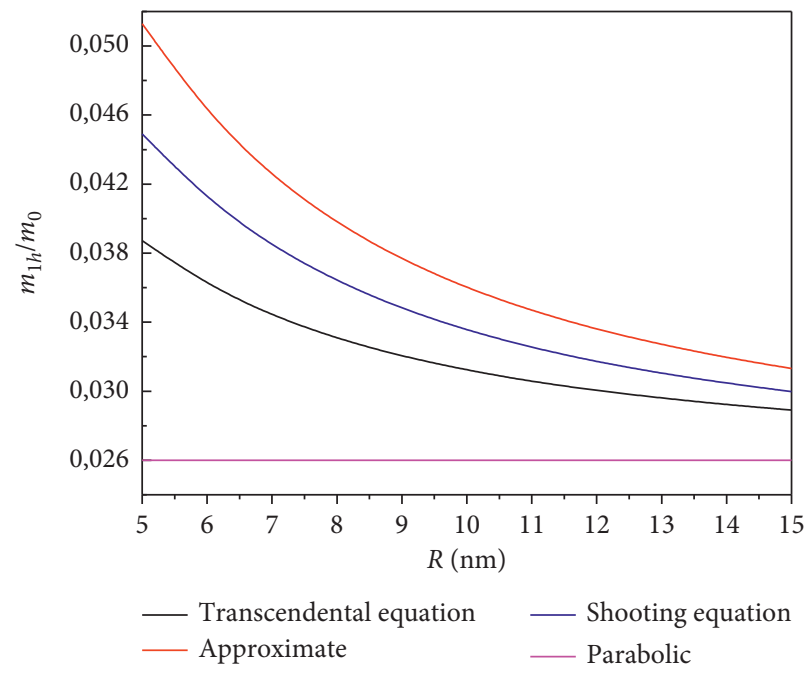

FIGURE 10: The dependence of the effective mass of a light hole on the radius of a cylindrical quantum wire with a finite potential.

distance inside the barrier is $R / 2$, and then the error in calculating the energy was $0.01 \mathrm{meV}$ compared to $\rho \longrightarrow \infty$. The dependence of the effective masses of electrons and holes on the radius of a cylindrical nanowire was determined (Figures 8-10). The results of the graphs show that the effective mass of charge carriers decreases with increasing radius of the nanowires and, at large radii, approaches $m_{0}$. Thus, the nonparabolicity of the system at small radii is more noticeable than at large radii.

The resulting expression (22) allows us to calculate the energy in cylindrical nanorod with inner and outer radii $R_{1}$ and $R_{2}$, respectively. The results are compared by expression (22) and expression (28) (Figures 11-13). Figure 14 shows the radial wave function of the electron. Here, we assumed 


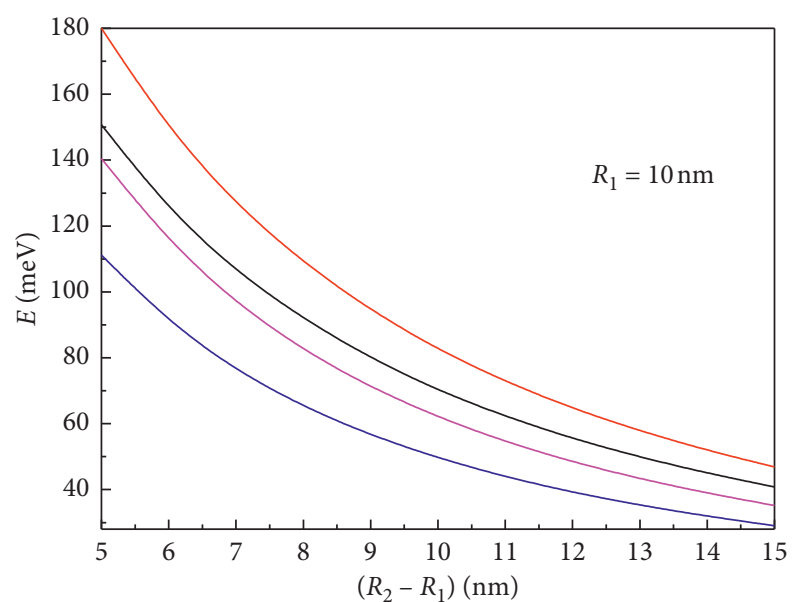

- Shooting equation, nonparabolic- $e 1$

— Shooting equation, parabolic-e 1

- Transcendental equation, nonparabolic-e 1

— Transcendental equation, parabolic- $e 1$

FIgURE 11: The dependence of the electron energy on the thickness of the layer of a cylindrical quantum nanorod with a finite potential.

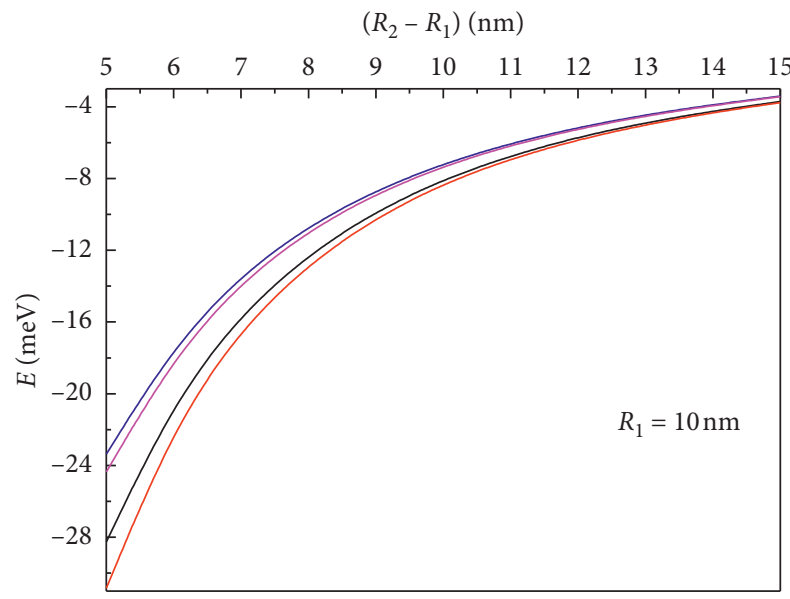

- Shooting equation, nonparabolic- $h h 1$

_ Shooting equation, parabolic- $h h 1$

- Transcendental equation, nonparabolic- $h h 1$

— Transcendental equation, parabolic- $h h 1$

FIGURE 12: The dependence of the energy of a heavy hole on the thickness of a layer of a cylindrical quantum nanorod with a finite potential.

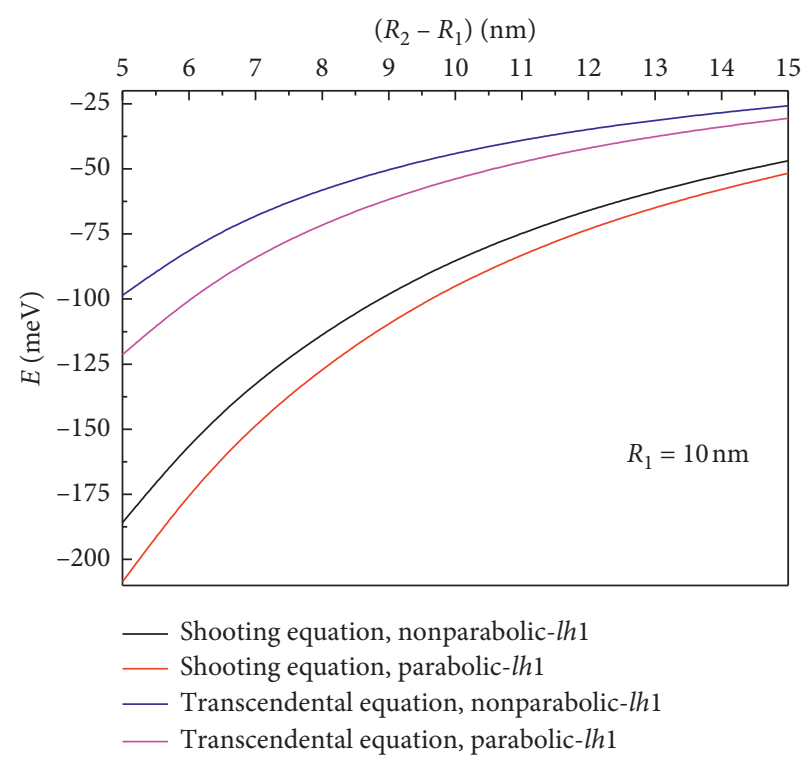

Figure 13: The dependence of the energy of a light hole on the layer thickness of a cylindrical quantum nanorod with a finite potential.

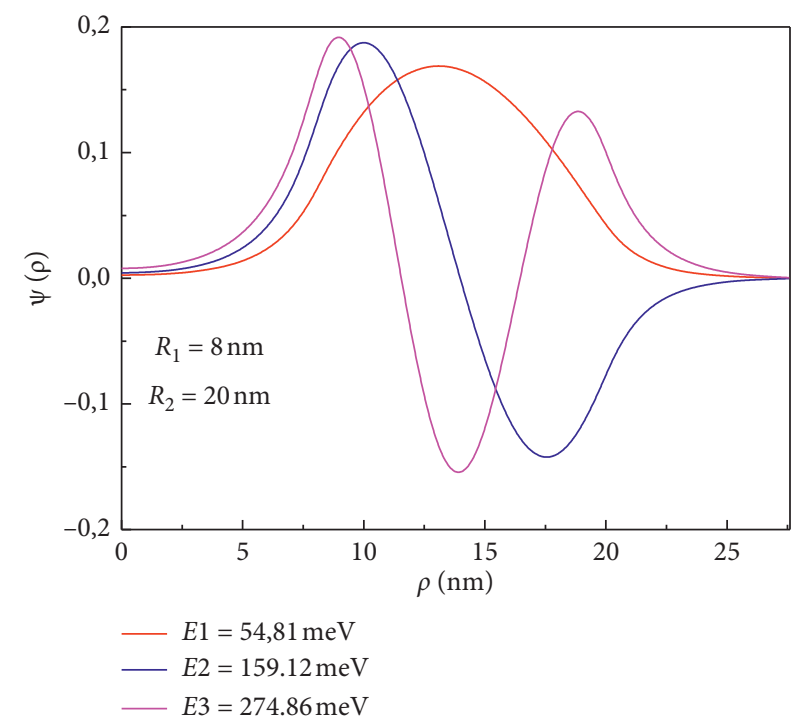

FIgURE 14: The dependence of the radial wave function of an electron on the radius of a cylindrical quantum nanorod with a finite potential. 


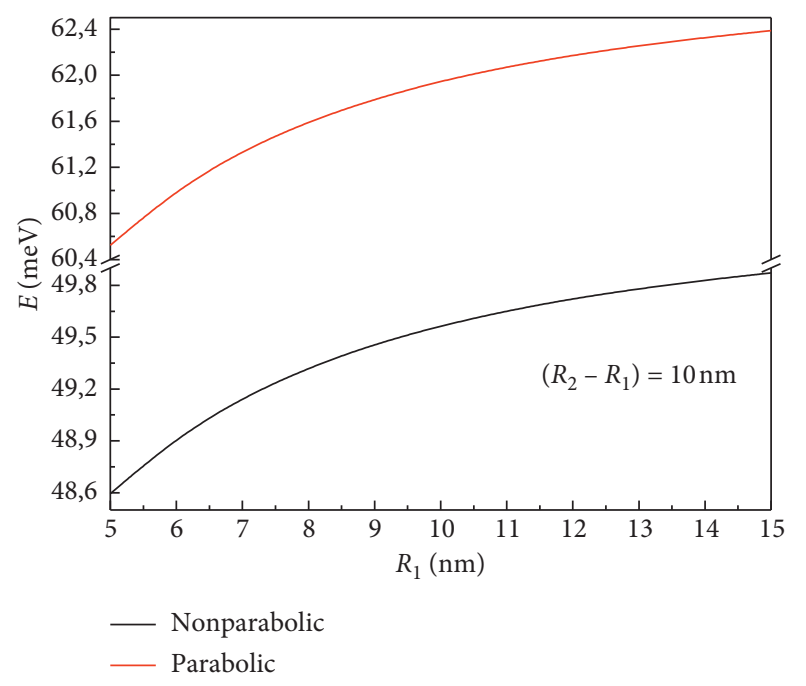

FIGURE 15: The dependence of the particle energy on the inner radius with a constant layer thickness of a cylindrical quantum nanorod and with a finite potential.

that the wave function inside the barrier at a distance $R_{1}$ is zero, and the error in this case is $0.01 \mathrm{meV}$. The results shown in Figure 15 allow us to draw the following conclusion: for a constant thickness of cylindrical nanorod, there is a weak dependence of the particle energy on the internal radius $R_{1}$, i.e., with an increase in $R_{1}$, a slight increase in particle energy is observed.

\section{Conclusion}

The Schrödinger equation for cylindrical nanowires and for cylindrical nanorods was solved by two methods. An approximate equation is obtained that determines the first energy level in cylindrical quantum wires with large radii. When solving the Schrödinger equation, the change in the effective masses of electrons and holes was taken into account. Graphs of the effective mass of electrons and holes versus nanowire radius are presented. The energy of electrons and holes in cylindrical nanorods was calculated, and the effects of parabolicity and nonparabolicity of the zones were compared. It is shown that, for a constant thickness of cylindrical nanorods, there is a weak dependence of the particle energy on the change in the internal radius $R_{1}$, that is, with an increase in $R_{1}$, a slight increase in the particle energy is observed.

\section{Data Availability}

The data that support the findings of this study are available on request from the corresponding author (Kh.N. Juraev).

\section{Conflicts of Interest}

The authors declare that they have no conflicts of interest.

\section{Acknowledgments}

This work was financially supported by the Program for Fundamental Research (grant number BA-FA-F-2-005).

\section{References}

[1] P. Mohan, J. Motohisa, and T. Fukui, "Controlled growth of highly uniform, axial/radial direction-defined, individually addressable InP nanowire arrays," Nanotechnology, vol. 16, no. 12, pp. 2903-2907, 2005.

[2] J. Motohisa, J. Noborisaka, J. Takeda, M. Inari, and T. Fukui, "Catalyst-free selective-area MOVPE of semiconductor nanowires on (111)B oriented substrates," Journal of Crystal Growth, vol. 272, no. 1-4, pp. 180-185, 2004.

[3] P. Mohan, J. Motohisa, and T. Fukui, "Realization of conductive InAs nanotubes based on lattice-mismatched InP / InAs core-shell nanowires," Applied Physics Letters, vol. 88, Article ID 013110, 2006.

[4] Y. I. Mazur, V. G. Dorogan, O. Bierwagen et al., "Spectroscopy of shallow InAs/InP quantum wire nanostructures," Nanotechnology, vol. 20, no. 6, 10 pages, Article ID 065401, 2009.

[5] B. Pal, K. Goto, M. Ikezawa et al., "Spectral diffusion of type-II excitons in wurtzite InP/InAs/InP core-multishell nanowires," Journal of Luminescence, vol. 129, no. 12, pp. 1941-1944, 2009.

[6] M. Bai, H. Huang, Z. Liu et al., "InAs/InP core/shell nanowire gas sensor: effects of InP shell on sensitivity and long-term stability," Applied Surface Science, vol. 498, p. 143756, 2019.

[7] Y. Li, K. Goto, P. Mohan, J. Motohisa, and T. Fukui, "Bimolecular interlayer scattering of electrons in InP/InAs/InP core-multishell nanowires," Journal of Luminescence, vol. 133, pp. 135-137, 2013.

[8] F. Tomimoto, M. Kammermeier, J. König et al., "Ultralong spin lifetimes in one-dimensional semiconductor nanowires," Applied Physics Letters, vol. 114, no. 20, p. 202101, 2019.

[9] E. O. Schüller, "Band structure of indium antimonide," Journal of Physics and Chemistry of Solids, vol. 1, no. 4, pp. 249-261, 1957.

[10] A. Godoy, Z. Yang, U. Ravaioli, and F. Gámiz, "Effects of nonparabolic bands in quantum wires," Journal of Applied Physics, vol. 98, Article ID 013702, 2005.

[11] F. M. Gómez-Campos, S. Rodríguez-Bolívar, and J. E. Carceller, "A new approach to analyzing anisotropic and non-parabolic effects on quantum wires," Journal of Computational Electronics, vol. 7, no. 3, pp. 342-345, 2008.

[12] B. M. Wong, F. Léonard, Q. Li, and G. T. Wang, "Nanoscale effects on heterojunction electron gases in GaN/AlGaN core/ shell nanowires," Nano Letters, vol. 11, no. 8, pp. 3074-3079, 2011.

[13] G. Gulyamov, A. G. Gulyamov, A. B. Davlatov, and B. B. Shahobiddinov, "Electron energy in rectangular and cylindrical quantum wires," Journal of Nano- and Electronic Physics, vol. 4, no. 04023, 2020.

[14] H. Sun, B.-C. Liu, and Q. Tian, "Polaron effects in cylindrical GaAs/Al x Ga1-x as core-shell nanowires," Chinese Physics B, vol. 26, no. 9, Article ID 097302, 2017.

[15] V. A. Harutyunyan, D. B. Hayrapetyan, and E. M. Kazaryan, "Optical transitions and photoluminescence in cylindrical core/layer/shell $\beta$-CdS $/ \beta$-HgS/ $\beta$-CdS heterostructure," Physics of the Solid State, vol. 62, no. 8, pp. 1305-1316, 2020.

[16] V. A. Harutyunyan, "Semiconductor nanotube in the field of uniformly charged ring: additional quantization in the form of one-dimensional hydrogen-type levels," Physica E: Low- 
Dimensional Systems and Nanostructures, vol. 57, pp. 69-75, 2014.

[17] S. Salimian, O. Arif, V. Zannier et al., "Electrical probing of carrier separation in InAs/InP/GaAsSb core-dualshell nanowires," Nano Research, vol. 13, no. 4, pp. 1065-1070, 2020.

[18] F. S. Ercolani, A. Baumgartner, L. Gubser et al., "Highly symmetric and tunable tunnel couplings in InAs/InP nanowire heterostructure quantum dots," Nanotechnology, vol. 31, no. 13, Article ID 135003, 2020.

[19] M. Cygorek, M. Korkusinski, and P. Hawrylak, "Atomistic theory of electronic and optical properties of InAsP/InP nanowire quantum dots," Physical Review B, vol. 101, no. 7, 2020.

[20] S. H. Ha and J. Zhu, "Temperature effect on shallow impurity states in a wurtzite GaN/Al Ga1-N core-shell nanowire," Physica E: Low-Dimensional Systems and Nanostructures, vol. 122, Article ID 114179, 2020.

[21] G. Liu, R. Liu, G. Chen, Z. Zhang, K. Guo, and L. Lu, "Nonlinear optical rectification and electronic structure in asymmetric coupled quantum wires," Results in Physics, vol. 17, Article ID 103027, 2020.

[22] M. Zieliński, "Vanishing fine structure splitting in highly asymmetric InAs/InP quantum dots without wetting layer," Scientific Reports, vol. 10, no. 1, p. 13542, 2020.

[23] A. Bouazra, S. Abdi-Ben Nasrallah, A. Poncet, Y. Bouazra, and M. Said, "Numerical simulation of coupling effect on electronic states in quantum wires," The European Physical Journal B, vol. 67, no. 2, pp. 245-250, 2009.

[24] A. Esposito, M. Luisier, M. Frey, and A. Schenk, "A nonparabolicity model compared to tight-binding: the case of square silicon quantum wires," Solid-State Electronics, vol. 53, no. 3, pp. 376-382, 2009.

[25] G. Shao-Wen, C. Jun-Cheng, and F. Song-Lin, "An emphasis of electron energy calculation in quantum wells," Communications in Theoretical Physics, vol. 42, no. 3, pp. 435-439, 2004.

[26] E. P. Pokatilov, V. A. Fonoberov, S. N. Balaban, and V. M. Fomin, "Electron states in rectangular quantum well wires (single wires, finite and infinite lattices)," Journal of Physics: Condensed Matter, vol. 12, no. 42, pp. 9037-9052, 2000.

[27] A. Jaffal, P. Regreny, G. Patriarche, N. Chauvin, and M. Gendry, "Density-controlled growth of vertical InP nanowires on Si (111) substrates," Nanotechnology, vol. 31, no. 35, Article ID 354003, 2020.

[28] R. Betancourt-Riera, R. Betancourt-Riera, L. A. Ferrer-Moreno, and A. D. Sañu-Ginarte, "Theory of electron Raman scattering in a semiconductor core/shell quantum well wire," Physica B: Condensed Matter, vol. 563, pp. 93-100, 2019.

[29] Ş. Aktas, F. K. Boz, A. Bilekkaya, and S. E. Okan, "The electronic properties of a coaxial square GaAs/AlxGa1-xAs quantum well wire in an electric field," Physica E: Low-Dimensional Systems and Nanostructures, vol. 41, no. 8, pp. 1572-1576, 2009.

[30] N. V. Tkach, I. V. Pronishin, and A. M. Makhanets, "Spectrum of an electron in a superlattice along a cylindrical quantum wire," Russian Physics Journal, vol. 41, no. 2, pp. 178-184, 1998.

[31] F. M. S. Lima, O. A. C. Nunes, A. L. A. Fonseca, M. A. Amato, and E. F. da Silva Jr, "Effect of a terahertz laser field on the electron-DOS in a GaAs/AlGaAs cylindrical quantum wire: finite well model," Semiconductor Science and Technology, vol. 23, no. 12, 6 pages, Article ID 125038, 2008.
[32] A. Radu, "Transverse laser dressing effects on the subband density of states in a $20 \mathrm{~nm}$-wide GaAs/Al0.3Ga0.7As quantum well wire," Physica E: Low-Dimensional Systems and Nanostructures, vol. 44, no. 7-8, pp. 1446-1453, 2012.

[33] B.R. Nag and S. Gangopadhyay, "Energy levels in quantum wires with finite barrier potential," Physica Status Solidi (A), vol. 179, p. 463, 1993.

[34] M. Tsetseri and G. P. Triberis, "A study of the ground state of quantum wires using the finite difference method," Superlattices and Microstructures, vol. 32, no. 1, pp. 79-90, 2002.

[35] A. Deyasi, S. Bhattacharyya, and N. R. Das, "A finite-difference technique for computation of electron states in core-shell quantum wires of different configurations," Physica Scripta, vol. 89, no. 6, p. 11, Article ID 065804, 2014.

[36] V. V. Arsoski, N. A. Čukarić, M. Ž. Tadić, and F. M Peeters, "An efficient finite-difference scheme for computation of electron states in free-standing and core-shell quantum wires," Computer Physics Communications, vol. 197, pp. 1726, 2004.

[37] M. Abramowitz and I.A. Stegun, Handbook of Mathematical Functions with Formulas, Graphs, and Mathematical Tables, Dover, New York, NY, USA, 10th edition, 1972.

[38] P. Harrison, Quantum Wells, Wires and Dots: Theoretical and Computational Physics of Semiconductor Nanostructures, John Wiley, Hoboken, NY, USA, 4th edition, 624 pages, John Wiley, Hoboken, NY, USA, 2005.

[39] J. Davies, Properties of Important Semiconductors. In The Physics of Low-dimensional Semiconductors: An Introduction, Cambridge University Press, Cambridge, UK, pp. 410-411, 1997.

[40] P. J. Baymatov and B. T. Abdulazizov, "Concentration dependences of the electron effective mass, fermi energy, and filling of subbands in doped InAs/AlSb quantum wells," Ukrainian Journal of Physics, vol. 62, no. 1, pp. 46-50, 2017.

[41] G. Bastard, Wave Mechanics Applied to Semiconductor Heterostructures, John Wiley \& Sons, New York, NY, USA, 1991.

[42] U. Ekenberg, "Nonparabolicity effects in a quantum well: Sublevel shift, parallel mass, and Landau levels," Physical Review B, vol. 40, no. 11, pp. 7714-7726, 1989.

[43] G. Gulyamov, U. I. Erkaboev, and A. G. Gulyamov, "Influence of pressure on the temperature dependence of quantum oscillation phenomena in semiconductors," Advances in Condensed Matter Physics, vol. 2017, Article ID 6747853, 6 pages, 2017.

[44] S. Adachi, "Material parameters of In1-xGaxAsyP1-yand related binaries," Journal of Applied Physics, vol. 53, no. 12, pp. 8775-8792, 1982.

[45] J. Robertson and B. Falabretti, "Band offsets of high K gate oxides on III-V semiconductors," Journal of Applied Physics, vol. 100, Article ID 014111, 2006.

[46] H. Q. Hart and C. W. Tu, "Optical property of InAsP/InP strained quantum wells grown on InP (111)Band (100) substrates," Journal of Applied Physics, vol. 75, no. 9, pp. 4673-4679, 1994.

[47] S. Saravanan and A. John Peter, "Phonon effects on interband optical transitions in InAs $0.8 \mathrm{P} 0.2$ /InP quantum wire," Journal of Luminescence, vol. 169, pp. 86-92, 2016.

[48] I. Lee, J. R. Meyer, and L. R. Ram-Mohan, "Band parameters for III-V compound semiconductors and their alloys," Journal of Applied Physics, vol. 89, no. 11, pp. 5815-5875, 2001.

[49] R. Leonelli, C. A. Tran, J. L. Brebner et al., "Optical and structural properties of metalorganic-vapor-phase-epitaxygrown InAs quantum wells and quantum dots in InP," Physical Review B, vol. 48, no. 15, pp. 11135-11143, 1993. 
[50] P. R. C. Kent and A. Zunger, "Biaxial strain-modified valence and conduction band offsets of zinc-blende GaN, GaP, GaAs, InN, InP, and InAs, and optical bowing of strained epitaxial InGaN alloys," Applied Physics Letters, vol. 81, no. 23, pp. 4377-4379, 2002. 